\title{
Study on Rub-Impact Dynamical Behavior of An Asymmetrical Rotor- Bearing with Variable Ratio of Stiffness
}

\author{
Tan Lian ${ }^{*}, 1$ and Dang Pei ${ }^{2}$ \\ ${ }^{1}$ North China University of Water Resources and Electric Power, Zhengzhou, 450011, China \\ ${ }^{2}$ Henan University of Technology, Zhengzhou, 450001, China
}

\begin{abstract}
A rub-impact mathematic model of an Asymmetrical rotor-bearing with nonlinear oil film force is built in the paper. The bifurcation diagrams of the response were given following the changing of ratio of stiffness. We analyzed the bifurcation and the chaos character of an Asymmetrical rotor-bearing with operating rotor stiffness changing, then we get the dynamical character and the law when the rubbing happen. The nonlinear dynamic behaviors of the system were studied by using the numerical value integral and Poincare mapping methods. By analysis the process of rub-impact, it is so complex and many low frequencies with large amplitude are in the range of $0.3 \sim 0.6 \mathrm{X}$. The way of period-chaos-period and the phenomena of diverging backward whirl are discovered when the rubbing happen. These results provide important theoretical references for the safety operation of generating sets and the exact identification of the faults in rotating machinery.
\end{abstract}

Keywords: Bifurcation, chaos, rotor, rubbing, stiffness ratio.

\section{INTRODUCTION}

Rotating machinery is widely used in industry site. In order to increase the performance of rotating machinery, the clearance between rotor-stator is designed to be smaller, rubbing more likely to occur. The rubbing can cause blade fracture, rotor instability, and even cause serious incidents. Therefore, it is necessary and important for early diagnosis of rotor fault to analyze the dynamical gap rotor. The stiffness ratio of the stator and disk is an important factor in leading to rubbing fault.

Rubbing fault is a typical model of nonlinear systems. The vibration spectrum is very complex [1]. The nonlinear oil film forces should be considered. The stiffness of a single contact between a rolling element and a race was obtained by the Hertzian contact theory, and the damping of a bearing was either artificially introduced to eliminate transient vibrations or provided by an approximate formula from Kramer [2].

Zhang et al. predicted the equivalent stiffness and damping coefficients at each rolling element contact using the transient EHL model and the free vibration model, but the results were not introduced to the analysis of dynamics of rotor-bearing systems [3].

To establish a reasonable model of the friction force and rotor is the key to solve the problem. The model of short bearings theory and the model of oil film proposed by Zhang Wen of the analytical expression are the same, and they are the simplified form of finite Explicit Model Proposed by Yang Jinfu [4-6]. In this paper, used the model of short bearings theory and the smooth friction force model with piecewise-linearity.

Rotor bearing system is a complex nonlinear system. With impact stiffness coefficient increased, the phenomenon of frequency division and chaos are induced more frequently. The numerical integration method of RungeKutta is used to obtain nonlinear dynamic responses of this system, and using time-domain waveform diagrams, the orbit diagrams of axle center, the frequency spectrogram, the Poincare maps, the bifurcation plots etc. [7, 8]. The impact of stiffness coefficient on system responses are studied.

\section{THE MODEL OF AN ASYMMETRICAL ROTOR- BEARING}

As the complexity of nonlinear rub-impact model, the rotor is simplified as elastic rotation shaft with concentrated weight. The model is established with nonlinear oil film force, and has rigid foundation and support. Fig. (1) shows the model.

The relationship between force and displacement of rotor and disk is nonlinear. The material properties and the shape of the contact surface decided the degree of nonlinearity. When the rubbing occurring, there is both the elastic and plastic deformation, and even mixed deformed state. Elastic deformation of the stress-strain relationship is linear, and the plastic deformation of the stress-strain relationship is nonlinear. So the contact force in normal direction and radial displacement has a non-linear relationship. Fig. (2) shows the model. 


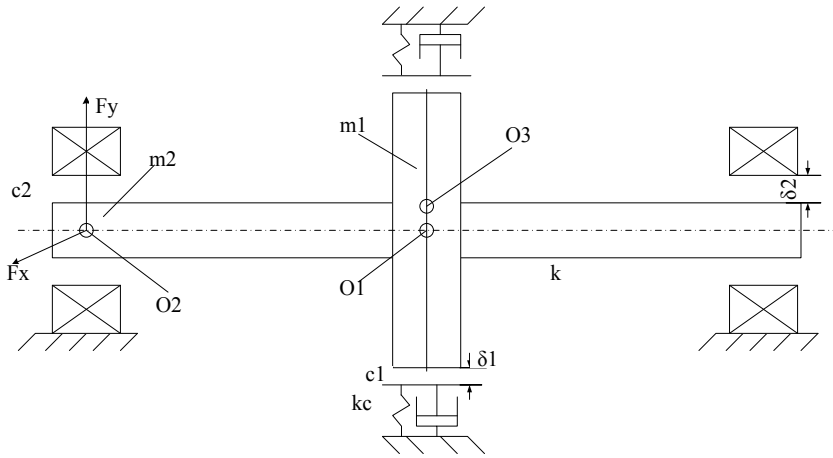

Fig. (1). Model of the rotor-bearing system.

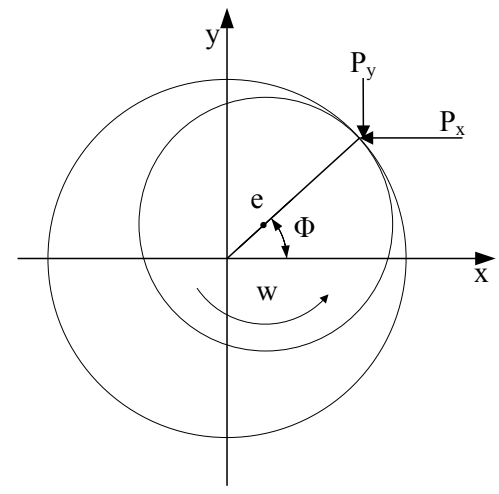

Fig. (2). The contact force and displacement.

The rotor is supported at both ends by sliding bearing. $O_{1}$ is the geometrical center of the rotor. $\mathrm{O}_{2}$ is the geometrical center of bearing bush. $\mathrm{O}_{3}$ is the weight center of the rotor. $\left(x_{1}, y_{1}\right)$ is center coordinates of the wheel, $\left(x_{2}, y_{2}\right)$ is center coordinates of axle journal, $k_{c}$ is the stiffness of stator. $k$ is the linear stiffness of elastic rotation shaft. $c_{1}$ is the damping coefficient of disk rotor. $c_{2}$ is the damping coefficient of the rotor on the sliding bearing. $\delta_{1}$ is the gap between the stator and disk rotor. $\delta_{2}$ is the gap between the stator and sliding bearing. $e$ is the eccentric weight of disk rotor. $2 m_{2}$ is the concentrated weight of the rotor on the sliding bearing. $m_{1}$ is concentrated weight of rotor on the disc. It is the elastic shaft without weight between the sliding bearing and the disk. $\mathrm{R}$ is the diameter of axle journal. $L$ is the length of the sliding bearing. $\omega$ is the rotation speed of rotor. $\varphi$ is the angular displacement of whirling angle. $\mathrm{D}$ is the radius of the sliding bearing.

Where, $D=2\left(R+\delta_{1}\right)$

\subsection{The Nonlinear Dynamic Equation of the System}

For the nonlinear dynamic equation of the system, the equation takes the following form:

$$
M \ddot{z}+D \dot{z}+K z=G+F_{z}+F_{r u b}
$$

where, $M$ is the weight matrix, $D$ is the damping matrix, $K$ is the stiffness matrix, $F(z)$ is the outside force, $G$ is the gravity vector, $F_{z}$ is the unbalanced force vector, $F_{r u b}$ is the rubbing force.

By analyzing the force of rotor disk in fixed coordinate system, the functions of disk and journal in horizontally and vertically direction is developed as follows:

$$
\begin{aligned}
& m_{1} x_{1}^{\prime \prime}+c_{1} x_{1}^{\prime}+\frac{1}{2}\left[\left(k_{1}+k_{2}\right)+\left(k_{1}-k_{2}\right) \cos (2 \omega t)\right]\left(x_{1}-x_{2}\right) \\
& +\frac{\left(k_{1}-k_{2}\right) \sin (2 \omega t)}{2}\left(y_{1}-y_{2}\right)=f_{x}+m_{1} \omega^{2} e \cos (\omega t) \\
& m_{1} y_{1}^{\prime \prime}+c_{1} y_{1}^{\prime}+\frac{1}{2}\left[\left(k_{1}+k_{2}\right)+\left(k_{1}-k_{2}\right) \cos (2 \omega t)\right]\left(y_{1}-y_{2}\right) \\
& +\frac{\left(k_{1}-k_{2}\right) \sin (2 \omega t)}{2}\left(x_{1}-x_{2}\right)=f_{y}+m_{1} \omega^{2} e \sin (\omega t)-m_{1} g \\
& m_{2} x_{2}^{\prime \prime}+\frac{c_{2}}{2} x_{2}^{\prime}+\frac{1}{4}\left[\left(k_{1}+k_{2}\right)+\left(k_{1}-k_{2}\right) \cos (2 \omega t)\right]\left(x_{2}-x_{1}\right) \\
& +\frac{\left(k_{1}-k_{2}\right) \sin (2 \omega t)}{4}\left(y_{2}-y_{1}\right)=p_{x} \\
& m_{2} y_{2}^{\prime \prime}+\frac{c_{2}}{2} y_{2}^{\prime}+\frac{1}{4}\left[\left(k_{1}+k_{2}\right)+\left(k_{1}-k_{2}\right) \cos (2 \omega t)\right]\left(y_{2}-y_{1}\right) \\
& +\frac{\left(k_{1}-k_{2}\right) \sin (2 \omega t)}{4}\left(x_{2}-x_{1}\right)=p_{y}-m_{2} g
\end{aligned}
$$

where, $P_{x}$ is the components of oil film force in horizontal direction, $P_{y}$ is the components of oil film force in vertical direction. $f_{x}$ is the radial rubbing force, $f_{y}$ is the tangential rubbing force.

\subsection{The Non-Dimensional Rubbing Force}

The rubbing force is decomposed as $f_{\mathrm{x}}$ and $f_{\mathrm{y}}$ by coulomb law of friction.

Taken $\hat{x}=x_{1} / \delta_{2}, \hat{y}=y_{1} / \delta_{2}$, the non-dimensionalization rubbing force in the $x-y$ Coordinate System could be acquired as follows:

$$
\left\{\begin{array}{l}
\left\{\begin{array}{l}
f_{\hat{x}_{1}} \\
f_{\hat{y}_{1}}
\end{array}\right\}=-\left(1-r_{\delta} / r\right) \frac{r_{c k}}{r_{m} \cdot \bar{\omega}^{2}}\left[\begin{array}{cc}
1 & -f \\
f & 1
\end{array}\right]\left\{\begin{array}{c}
\hat{x}_{1} \\
\hat{y}_{1}
\end{array}\right\}, r \geq r_{\delta} \\
\left\{\begin{array}{l}
f_{\hat{x}_{1}} \\
f_{\hat{y}_{1}}
\end{array}\right\}=\left[\begin{array}{ll}
0 & 0 \\
0 & 0
\end{array}\right]\left\{\begin{array}{l}
\hat{x}_{1} \\
\hat{y}
\end{array}\right\}
\end{array}\right.
$$

where,

$$
r=\sqrt{\hat{x}_{1}^{2}+\hat{y}_{1}^{2}}, r_{c k}=k_{c} / k_{2}, r_{\delta}=\delta_{1} / \delta_{2}
$$

$f$ is the friction coefficient, $r$ is the radial displacement of disc rotor. 


\subsection{The Nonlinear Oil Film Force After Non- Dimensionalization}

In the rotor-sliding bearing system, because the perturbations of axle journal, lubricant in the bearing will be rotated and formed the oil film, then the bracing force hold up the rotor. All the model of oil force is based on Reynolds equation and the pressure on the boundary film is zero. Capone proposed a model about nonlinear oil-film force on the infinitely short journal bearing in 1991, the calculating results shows better accuracy and convergence.

The non-dimensional force is the following function:

$\left[\frac{r_{0}}{L}\right]^{2} \frac{\partial}{\partial z}\left[h^{3} \frac{\partial p}{\partial z}\right]=x \sin \varphi-y \cos \varphi-2\left(x^{\prime} \cos \varphi+y^{\prime} \sin \varphi\right)$

We can get the formula as follows by decomposed to radial direction and axis direction.

$\left\{\begin{array}{l}p_{\hat{x}}=K(3 \hat{x} \cdot V(\hat{x}, \hat{y}, \alpha)-\sin \alpha \cdot G(\hat{x}, \hat{y}, \alpha)-2 \cos \alpha \cdot S(\hat{x}, \hat{y}, \alpha)) \\ p_{\hat{y}}=K(3 \hat{y} \cdot V(\hat{x}, \hat{y}, \alpha)+\cos \alpha \cdot G(\hat{x}, \hat{y}, \alpha)-2 \sin \alpha \cdot S(\hat{x}, \hat{y}, \alpha))\end{array}\right.$

where,

$$
\begin{aligned}
& K=\frac{\sqrt{\left(\hat{x}-2 \hat{y}^{\prime}\right)^{2}+\left(\hat{y}+2 \hat{x}^{\prime}\right)^{2}}}{1-\hat{x}^{2}-\hat{y}^{2}} \\
& V(\hat{x}, \hat{y}, \alpha)=\frac{2+(\hat{y} \cos \alpha-\hat{x} \sin \alpha) \cdot G(\hat{x}, \hat{y}, \alpha)}{1-\hat{x}^{2}-\hat{y}^{2}} \\
& S(\hat{x}, \hat{y}, \alpha)=\frac{\hat{y} \sin \alpha+\hat{x} \cos \alpha}{1-(\hat{y} \sin \alpha+\hat{x} \cos \alpha)^{2}} \\
& G(\hat{x}, \hat{y}, \alpha)=\frac{2}{1-\hat{x}^{2}-\hat{y}^{2}}\left[\frac{\pi}{2}+\arctan \frac{\hat{y} \cos \alpha-\hat{x} \sin \alpha}{\sqrt{1-\hat{x}^{2}-\hat{y}^{2}}}\right] \\
& \alpha=\arctan \frac{\hat{y}+2 \hat{x}^{\prime}}{\hat{x}-2 \hat{y}^{\prime}}-\frac{\pi}{2} \operatorname{sign}\left(\frac{\hat{y}+2 \hat{x}^{\prime}}{\hat{x}-2 \hat{y}^{\prime}}\right)-\frac{\pi}{2} \operatorname{sign}\left(\hat{y}+2 \hat{x}^{\prime}\right)
\end{aligned}
$$

\subsection{The Non-Dimensional Nonlinear Dynamic Equation of the System}

Taken formula (3) and formula (4) into the formula (2). Make

$b=r / c, \hat{x}_{1}=x_{1} / c, \hat{y}_{1}=y_{1} / c, \hat{x}_{1}^{\prime}=x_{1}^{\prime} / \omega c, \hat{y}_{1}^{\prime}=y_{1}^{\prime} / \omega c$,

$\hat{x}_{1}^{\prime \prime}=x_{1}^{\prime \prime} / \omega^{2} c, \hat{y}_{1}^{\prime \prime}=y_{1}^{\prime \prime} / \omega^{2} c, \hat{x}_{2}=x_{2} / c, \hat{y}_{2}=y_{2} / c$, $\hat{x}_{2}^{\prime}=x_{2}^{\prime} / \omega c, \hat{y}_{2}^{\prime}=y_{2}^{\prime} / \omega c, \hat{x}_{2}^{\prime \prime}=x_{2}^{\prime \prime} / \omega^{2} c, \hat{y}_{2}^{\prime \prime}=y_{2}^{\prime \prime} / \omega^{2} c$, $r_{m}=m_{1} / m_{2}, \quad r_{k}=k_{1} / k_{2}, \rho=e / \delta_{2}, \quad \xi_{1}=c_{1} /\left(m_{1} \omega\right)$, $\xi_{2}=c_{2} /\left(m_{2} \omega\right), r_{\delta}=\delta_{1} / \delta_{2}, f_{0}=\sqrt{k_{2} / m_{2}}, \bar{\omega}=\omega / f_{0}$.

We can get the movement differential formula shows as follows:

$$
\begin{aligned}
& \hat{x}_{1}^{\prime \prime}+\zeta_{1} \hat{x}_{1}^{\prime}+\frac{\left(r_{k}+1\right)+\left(r_{k}-1\right) \cos 2 \tau}{r_{m} \cdot 2 \bar{\omega}^{2}}\left(\hat{x}_{1}-\hat{x}_{2}\right) \\
& +\frac{\left(r_{k}-1\right) \sin 2 \tau}{r_{m} \cdot 2 \bar{\omega}^{2}}\left(\hat{y}_{1}-\hat{y}_{2}\right)=\rho \cos \tau+f_{x} \\
& \hat{y}_{1}^{\prime \prime}+\zeta_{1} \hat{y}_{1}^{\prime}+\frac{\left(r_{k}+1\right)+\left(r_{k}-1\right) \cos 2 \tau}{r_{m} \cdot 2 \bar{\omega}^{2}}\left(\hat{y}_{1}-\hat{y}_{2}\right) \\
& +\frac{\left(r_{k}-1\right) \sin 2 \tau}{r_{m} \cdot 2 \bar{\omega}^{2}}\left(\hat{x}_{1}-\hat{x}_{2}\right)=\rho \sin \tau+f_{y}-G \\
& \hat{x}_{2}^{\prime \prime}+\frac{\zeta_{2}}{2} \hat{x}_{2}^{\prime}+\frac{\left(r_{k}+1\right)+\left(r_{k}-1\right) \cos 2 \tau}{4 \bar{\omega}^{2}}\left(\hat{x}_{2}-\hat{x}_{1}\right) \\
& +\frac{\left(r_{k}-1\right) \sin 2 \tau}{4 \bar{\omega}^{2}}\left(\hat{y}_{1}-\hat{y}_{2}\right)=\frac{S P_{x}\left(\hat{x}_{2}, \hat{y}_{2}\right)}{\bar{\omega}^{2} f_{0}^{2} m_{2} \sigma_{2}} \\
& \hat{y}_{2}^{\prime \prime}+\frac{\zeta_{2}}{2} \hat{y}_{2}^{\prime}+\frac{\left(r_{k}+1\right)+\left(r_{k}-1\right) \cos 2 \tau}{4 \bar{\omega}^{2}}\left(\hat{y}_{2}-\hat{y}_{1}\right) \\
& +\frac{\left(r_{k}-1\right) \sin 2 \tau}{4 \bar{\omega}^{2}}\left(\hat{x}_{2}-\hat{x}_{1}\right)=\frac{S P_{y}\left(\hat{x}_{2}, \hat{y}_{2}\right)}{\bar{\omega}^{2} f_{0}^{2} m_{2} \sigma_{2}}-G
\end{aligned}
$$

where, $\quad \tau=\omega t, \quad \omega$ is the angular speed of rotor, $G=g /\left(\omega^{2} c\right)$ is the nondimensional gravitation. $\mathrm{S}$ is the Sommerfeld-correction coefficient, the expression shows as follows:

$$
s=\frac{u \omega R L}{M}\left(\frac{R}{c}\right)^{2}\left(\frac{L}{2 R}\right)^{2}
$$

\section{RESULTS}

Parameters of the system are picked up as follows:

$$
\begin{aligned}
& m_{1}=420 \mathrm{~kg}, \quad m_{2}=50 \mathrm{~kg}, \quad R=14 \mathrm{~mm}, \quad L=12 \mathrm{~mm}, \\
& \delta_{2}=0.2 \mathrm{~mm}, \quad u=0.018 \mathrm{~Pa} \cdot \mathrm{s}, \quad c_{1}=4300 \mathrm{~N} \cdot \mathrm{s} / \mathrm{m}, \\
& c_{2}=9300 \mathrm{~N} \cdot \mathrm{s} / \mathrm{m}, \quad r_{\delta}=1.2, \quad r_{c k}=10, \quad \rho=0.08, \\
& \omega=650 \mathrm{rads}^{-1}, \bar{\omega}=0.2 .
\end{aligned}
$$

The numerical integration method of Runge-Kutta is used to obtain nonlinear dynamic responses of this system by Matlab. Making the time dimensionless, and integral step selection $\Delta t=1 / 1024$. In order to eliminate the negative effects of instantaneous response, the solution of the system to initial conditions were obtained in 1200 periods and only hold a restricted set of values in last 100 periods.

From the bifurcation diagram, the vibrant characteristic, however, is a complicated process. And it can be divided into two processes: rubbing and the stage without rubbing. 


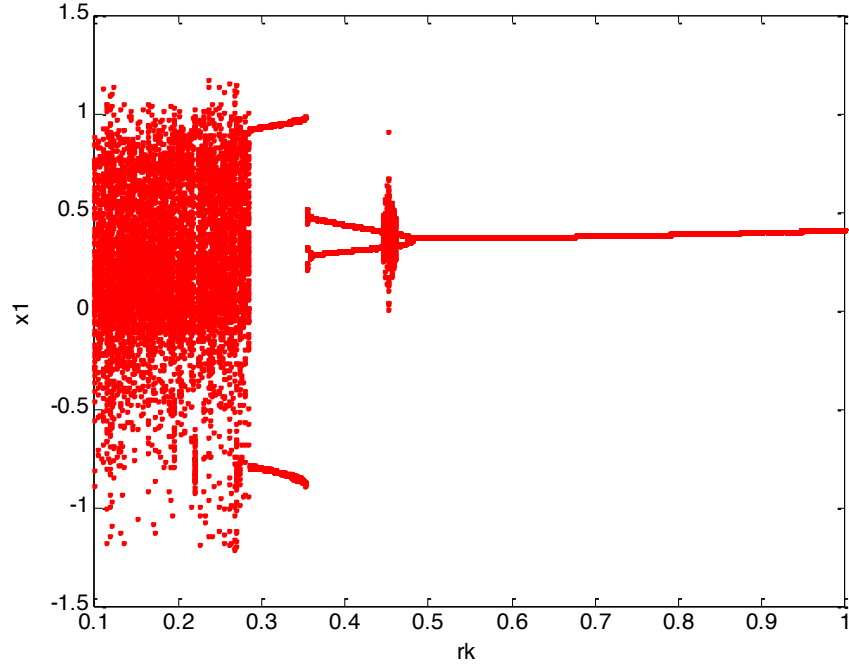

Fig. (3). The bifurcation diagram in $\mathrm{x}_{1}$.

\subsection{The Stage Without Rubbing}

For the range $0.28 \leq r_{k} \leq 1$, the rotor with low difference of axial stiffness and radial stiffness, the rotor and the stator is without rub impact. The vibrant characteristic shown in Fig. (3). is the response of the system caused by mass eccentricity and nonlinear oil film force.

For the range $0.48 \leq r_{k} \leq 1$, the system is in the P-1 motion form. Fig. (4). shows the vibration waveform of rotor vibration, the axis orbit of the rotor disc, the spectrogram of the vibration waveform and the Poincare maps when $r_{k}=0.6$. There is only one fixed points in the Poincare map, it shows that the system is in the P-1 movement. Spectrogram of the vibration waveform has only basic frequency. The range of the rotor movement is on the small side in $\mathrm{x}-\mathrm{y}$ direction.
When $r_{k}$ changes from 0.48 to 0.26 , the range of the rotor movement increases with the increasing of difference of stiffness in axial direction and radial direction. The vibrant characteristic went through three steps: P-2 movement, quasi-period movement, chaotic movement.

Fig. (5) shows the vibration waveform of rotor vibration, the axis orbit of the rotor disc, the spectrogram of the vibration waveform and the Poincare maps when $r_{k}=0.48$. Spectrogram of the vibration waveform has half-frequency, basic frequency, third - twos frequency and thrice frequency. Half-frequency shows the oil film whirl slightly. There are two fixed points in the Poincare map, it shows that the system is in the P-2 motion form.

When $r_{k}=0.44$, Fig. (6) shows the vibration waveform of rotor vibration, the axis orbit of the rotor disc, the spectrogram of the vibration waveform and the Poincare maps. Spectrogram of the vibration waveform has halffrequency, basic frequency, two-thirds frequency. Halffrequency and two-thirds frequency, caused by nonlinear oil film force, shows the oil film whirl seriously. There are two close curves in the Poincare map, it shows that the system is in the quasi-period movement.

Form Fig. (7), we can see that the system is in chaotic movement. Spectrogram of the vibration waveform has more low-frequency and less basic frequency. Many low frequencies with large amplitude are in the range of $0.3 \sim 0.6$ $X$. The increasing of low-frequency and the axis orbit of the rotor disc show that oil-film whirl of sliding bearing exacerbates the problem. The Poincare map shows that the system is in the quasi-period movement.

By the decreasing of $r_{k}$ from 0.44 to 0.26 , the system changes for chaotic movement to period movement. The vibration of the rotor disk gets worse, and is close to rubbing. Fig. (8). shows the system is in P-2 movement. Fig. (9) shows the system is in P-4 movement. And the halffrequency caused by nonlinear oil film force is increasing.
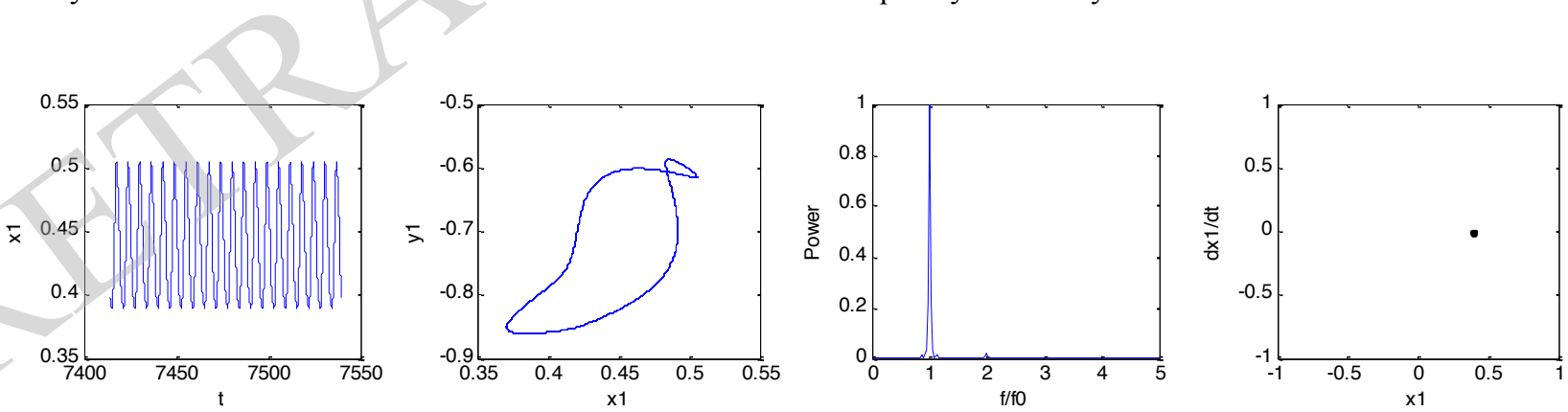

Fig. (4). The times-history, Poincare maps, Spectrogram of the vibration waveform, axes contrails of $r_{k}=0.6$.
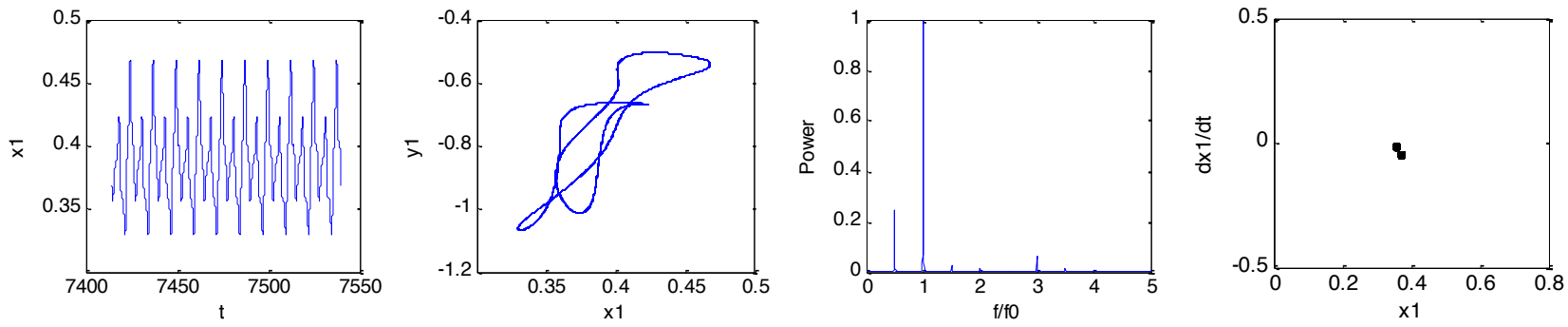

Fig. (5). The times-history, Poincare maps, Spectrogram of the vibration waveform, axes contrails of $r_{k}=0.48$. 

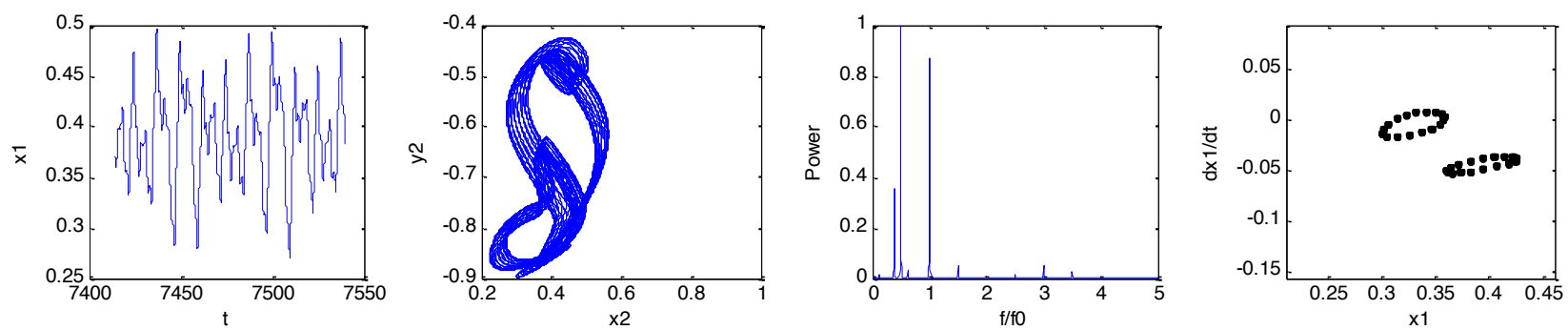

Fig. (6). The times-history, Poincare maps, Spectrogram of the vibration waveform, axes contrails of $r_{k}=0.46$.
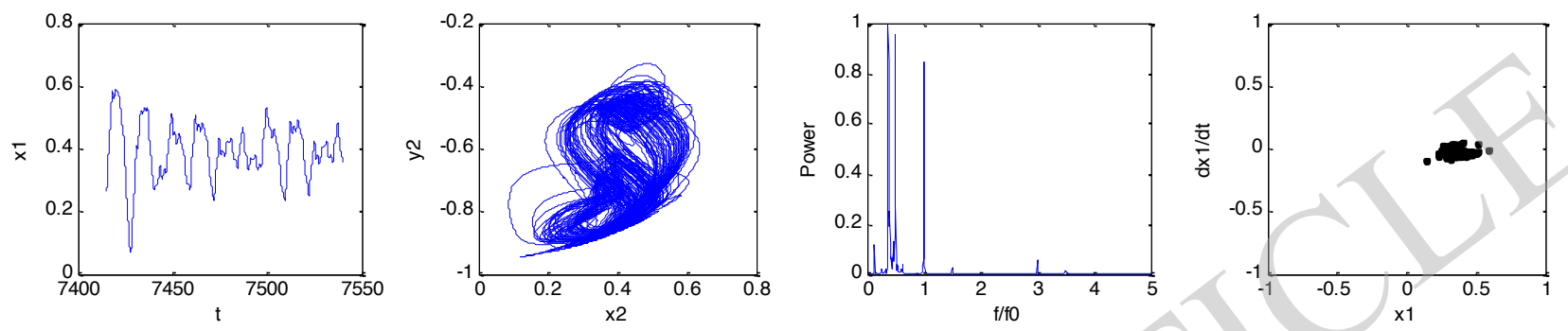

Fig. (7). The times-history, Poincare maps, Spectrogram of the vibration waveform, axes contrails of $r_{k}=0.45$
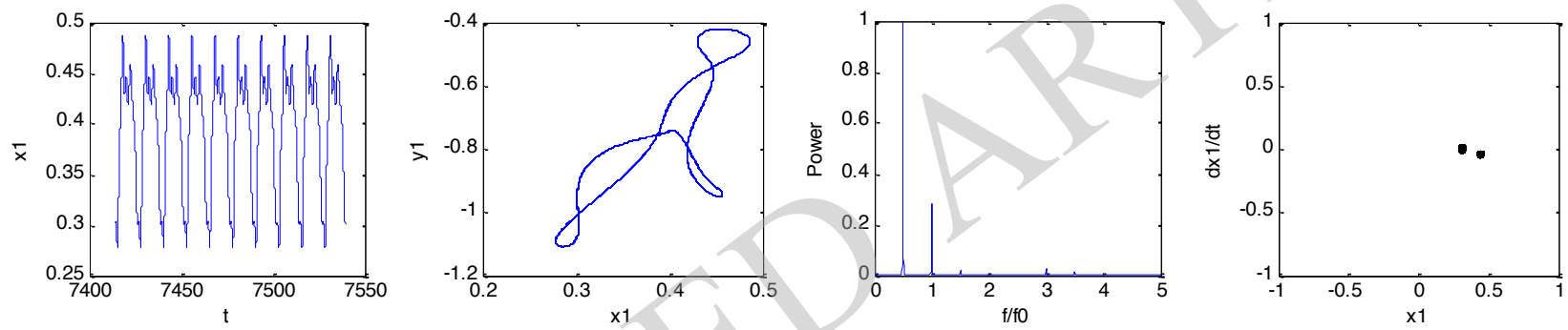

Fig. (8). The times-history, Poincare maps, Spectrogram of the vibration waveform, axes contrails of $r_{k}=0.4$.
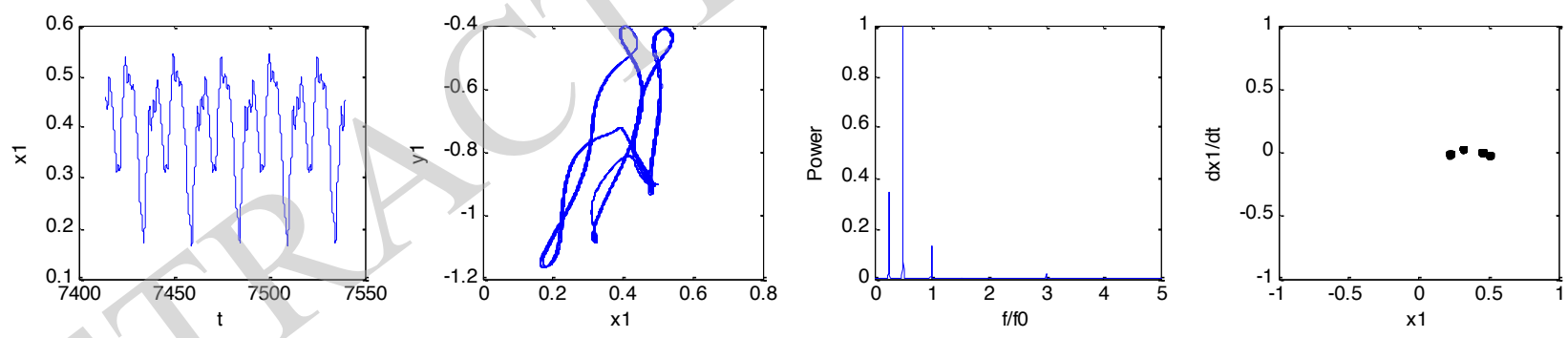

Fig. (9). The times-history, Poincare maps, Spectrogram of the vibration waveform, axes contrails of $r_{k}=0.35$.
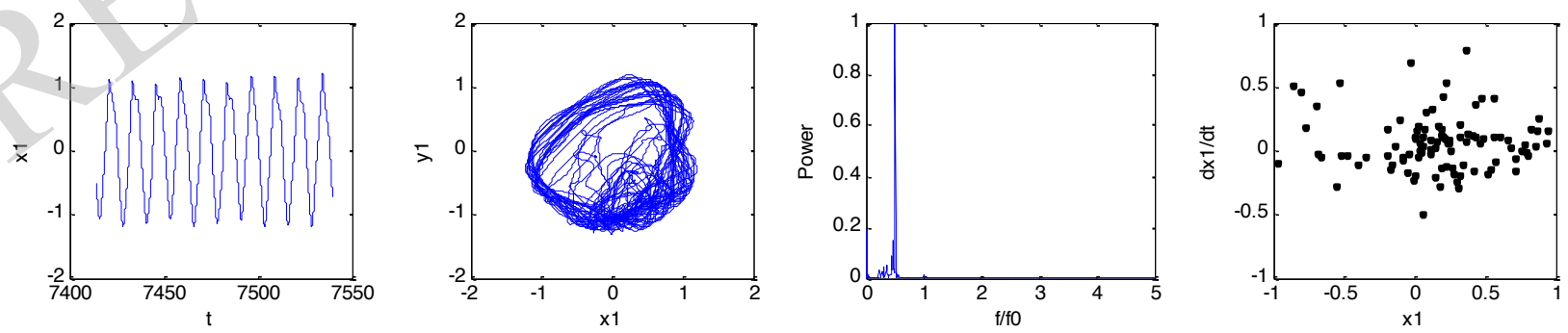

Fig. (10). The times-history, Poincare maps, Spectrogram of the vibration waveform, axes contrails of $r_{k}=0.25$.

\subsection{Rubbing Stage}

The system is moved in the chaotic movement by the decreasing of $r_{k}$. And it caused rubbing impact. The rubbing impact caused the oil film whirl worse and increased the low- frequency. The low frequencies with large amplitude are in the range of $0.2 \sim 0.5 \mathrm{X}$. It was shown in Figs. $(\mathbf{1 0}, \mathbf{1 1})$. 

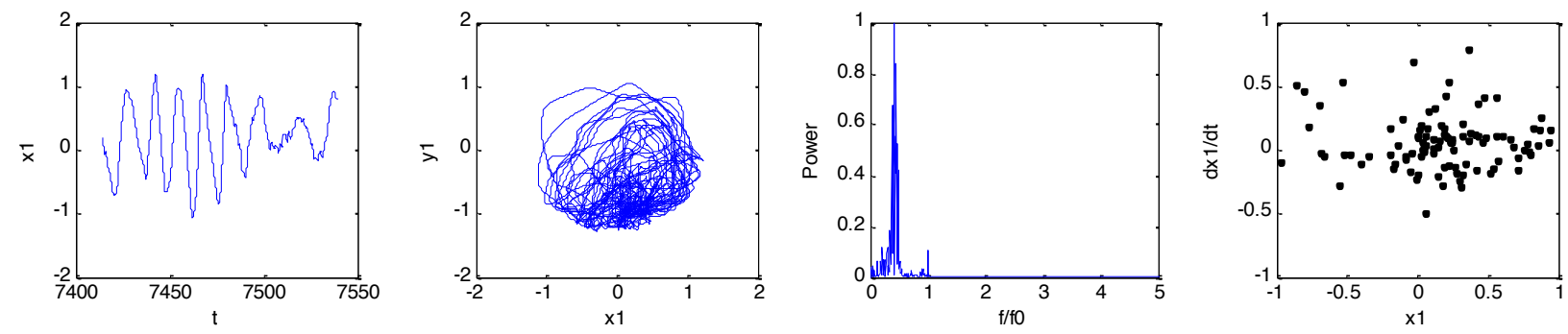

Fig. (11). The times-history, Poincare maps, Spectrogram of the vibration waveform, axes contrails of $r_{k}=0.15$.

\section{CONCLUSION}

Dynamic model of nonlinear rigidity-rotor system with nonlinear stiffness and nonlinear oil film force was set up. A rub-impact dynamical behavior of an Asymmetrical rotorbearing with nonlinear oil film force is studied. We analyzed the bifurcation and the chaos character of an Asymmetrical rotor-bearing. And the effect of asymmetry of rotor stiffness on dynamical character of the system, then we gain the dynamical character and the law when the rubbing happen. Considering asymmetry of rotor stiffness, we get the bifurcation diagram in using stiffness ratio $r_{k}$ as parameter. The way of period-chaos-period and the phenomena of diverging backward whirl are discovered when the rubbing happen.

The movement of the system can be divided into two processes: rubbing and the stage without rubbing, by changing $r_{k}$. The system is in the stage without rubbing, when it has high ratios of $r_{k}$. By the decrease of $r_{k}$, the system changes from periodic motion to $\mathrm{p}-\mathrm{K}$ periodic motion, eventually into a chaotic state shortly. At the same time, it enlarges the range of the disk movement and the value of low-frequency. The system is in rubbing stage when it has low ratios of $r_{k}$. Lower ratios of $r_{k}$ cause oil film whirl more seriously. The system is in chaotic movement.

\section{CONFLICT OF INTEREST}

The authors confirm that this article content has no conflict of interest.

\section{ACKNOWLEDGEMENTS}

Declared none.

\section{REFERENCES}

[1] C. He, and L. Yang, "Influence of rotating speed on the coupled bending and torsional vibrations of turbo-generator unit with rubimpact", Int. J. Online Eng., vol. 9, pp. 26-30, 2013.

[2] E. Kramer, Dynamics of Rotors and Foundations, Berlin: SpringerVerlag, 1993.

[3] Y.Y. Zhang, X.L. Wang, and X.L. Yan, "Dynamic behaviors of the elastohydrodynamic lubricated contact for rolling bearings", $A S M E$ J. Tribol., vol. 135, no. 2, pp. 021501, 2013.

[4] Y. Chen, H. Zhang, "Review and prospect on the research of dynamics of complete aero-engine systems", Hangkong Xuebao, vol. 8, pp. 1371-1391, 2011.

[5] W. Zhang, The Theoretical Basis of Rotor Dynamics, Beiking: Science Press, pp. 163-189, 1990.

[6] C.Li, H. Li, H. Ma, B. Wen, "Bifurcation and stability of the flexible rotor-bearing system with rub-impact by a continuum model", J. Mech. Eng., vol. 146, pp. 107-113, 2010.

[5] C.L. Liu, Ch. M. Xia, J.R. Zheng, B. Ch. Wen, "On the bifurcation of periodic motion of rotor system with rub-impact and oil fault", $J$. Vib. Shock, vol. 27, pp. 85-88, 2008.

[6] L. Tian, W. J. Wang, Z. J. Peng, "Nonlinear effects of unbalance in the rotor-floating ring bearing system of turbochargers", Mech. Syst. Signal Proce., vol. 34, pp. 298-320, 2013.

[7] X. F. Xu, W. Zhang, "Bifurcation and chaos of rigid unbalance rotor in short bearings under an unsteady oil-film force model", $J$. Vib. Eng., vol. 13, no, 2, pp. 247-253, 2000.

[8] S.H. Zhang, Y.G. Luo, B. Wu, H.Y. Hu, "Nonlinear response analysis of three-span rotor-bearing system with three cracks in shafts", Adv. Mat. Res., TTP, vol. 753-755, pp. 1093-1097, 2013. 